\title{
Medical treatment of diabetic retinopathy
}

\author{
Abstract \\ Eye (2003) 17, 550-562. doi:10.1038/ \\ sj.eye. 6700586
}

\section{Introduction}

It is well remembered that Canadians, Frederick Banting and John Mcleod, jointly received the 1923 Nobel Prize in Medicine for the discovery of insulin, and that Eli Lilly, whose researchers collaborated with Banting, began the first commercial manufacture of purified insulin under the trade name 'Iletin' in the same year. Since that time, the retinal microvascular complications of diabetes have become the major cause of blindness in the work age population throughout the Western world. Furthermore, the continued collaboration of clinicians and the pharmaceutical industry has led to considerable progress in the understanding and prevention of diabetic retinopathy: by the trialling of specific interventions generated by research into the pathogenesis of microvascular disease and as derivative data gained from large medical treatment trials.

Laser photocoagulation has been the mainstay of treatment of proliferative diabetic retinopathy and also of maculopathy for three decades. In 1970, The DRS reported that panretinal photocoagulation reduced the incidence of severe visual loss by $50 \%$ over 5 years in eyes with proliferative diabetic retinopathy. ${ }^{1}$ In 1985, The ETDRS reported the efficacy of focal laser photocoagulation for the treatment of clinically significant diabetic maculopathy and showed a $50 \%$ decrease in the incidence of moderate visual loss at 5 years. ${ }^{2}$ While landmark studies form the basis of the current treatment of diabetic retinopathy, the search has been for medical therapies to prevent or ameliorate progression of diabetic retinopathy. Biochemical explanations for the toxicity of glucose in diabetes have led to trials of specific medical therapies, which, on theoretical grounds, might afford cellular protection from damage owing to hyperglycaemia and thereby decrease diabetic retinopathy. Aspirin ${ }^{3,4}$ and Sorbinil ${ }^{5}$ are the most well known of these and none, or only minor benefits have been shown after much investigation. Several others have also undergone clinical evaluation and have been disappointing. Nevertheless, novel therapies for the prevention of diabetic complications are currently undergoing clinical evaluation (see Figure 1). However, confirming the benefit of existing therapies (hypoglycaemic and antihypertensive treatment) has come from recent landmark studies, which have confirmed good glycaemic control slows the development of retinopathy (UKPDS ${ }^{6}$ and $\mathrm{DCCT}^{7}$ ) and have also demonstrated that drugs currently in use for controlling blood pressure have clinically substantial retinoprotective effect in diabetes (UKPDS, ${ }^{8}$ EUCLID, ${ }^{9}$ and MICROHOPE ${ }^{10}$ studies).

\section{Pathogenesis}

There is no single explanation for the toxicity of glucose in the setting of deranged glucose control. Loss of control gives rise to a fluctuating blood glucose level with periods of hyperglycaemia. As a consequence of the loss of glucose control, metabolic and physiologic abnormalities, which are still being clarified, occur in the retina and the blood (see Figure 2). These lead to capillary cell death, alteration of retinal pericyte/endothelial cell ratio, and vascular occlusion. ${ }^{11}$ The resultant ischaemia releases growth factors that give rise to neovascularisation and increased vascular permeability. ${ }^{11}$ Sight-threatening pathological changes are strongly related to leakage and development of ischaemia in the retinal tissue, which leads to maculopathy and proliferative diabetic retinopathy, respectively.

\section{Sorbitol}

In 1959, Van Heyningen published in Nature that an excess of sorbitol was found in the lenses 
- ANTIOXIDANTS

- STATINS AND FIBRATES

- ANGIOTENSIN 11 RECEPTOR BLOCKADE

- PKC INHIBITION

- GROWTH HORMONE REDUCTION

- GLYCOSYLATION INHIBITION

Figure 1 Medical treatments for retinopathy.

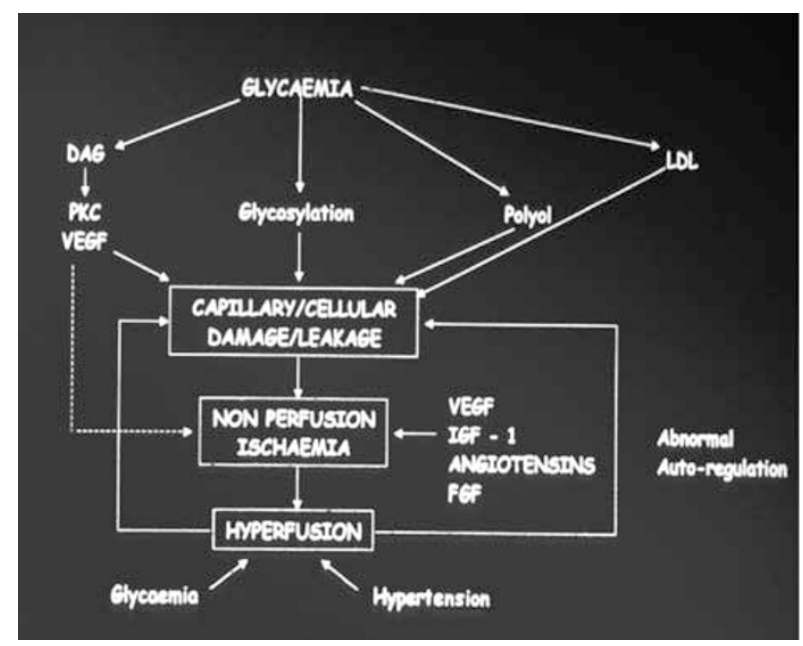

Figure 2 Pathogenetic mechanisms of diabetic retinopathy. DAG, diacylglycerol; PKC, protein kinase C pathway; VEGF, vascular endothelial growth factor; IGF-1, insulin growth factor 1; FGF, fibroblast growth factor.

of diabetic rats. ${ }^{12}$ The theory was elaborated that osmotic stress caused by the accumulation of sorbitol through the aldose reductase pathway in conditions of

hyperglycaemia was a mechanism of cellular injury in diabetes. This was not to be the case in humans. In a major review of the role of aldose reductase in diabetes, Crabbe and Goode showed that the rat model for cataract formation is inappropriate for human comparison, because of much higher ratio of polyol dehydrogenase to aldose reductase in the human lens. ${ }^{13}$ They concluded that based on current knowledge it was difficult to see any role for osmotic damage as an initiating factor in human diabetic complications. Aldose reductase appears to be part of a cellular osmotic defense system with accumulation of sorbitol occurring intracellularly in tissues, which are challenged by increased osmotic load. Excess utilisation of NADPH by aldose reductase in conditions of hyperglycaemia may limit access to this cofactor by NO synthetase and glutathione peroxidase and provide a rationale for the development of diabetic complications on the basis that competition for NADPH may stress antioxidant defences. ${ }^{13}$

Despite the doubt that now surrounds the theory of osmotic stress, clinical trials of aldose reductase inhibitors (ADI) have shown benefits and have been approved for use in some countries. There are nine aldose reductase inhibitors that have undergone clinical assessment and these are tolrestat, ponalrestat, zenarestat, epalrestat, sorbinil, zopolrestat, symmetristat, and ADN138. The spectrum of action of ADIs is not limited to inhibiting aldose reductase. The NAPDH sparing effect of ADIs is thought to act by a mechanism that is independent of aldose reductase. Much of the clinical research of ADIs has been on patients with peripheral neuropathy. Increases in nerve conduction rates, blood flow, and vasodilation have been demonstated with ADIs. ${ }^{13}$

With regard to retinal studies, the rate of new microaneurysm formation was slightly reduced in a single long-term study of sorbinil ${ }^{5}$ but the preventative effects on retinopathy seem less strong than in normoglycaemic trials of similar duration. A 6-month randomised study of tolrestat involving 31 patients with retinopathy showed a significant decrease in the rate of leakage from focal lesions on fluorescein angiography. ${ }^{14}$ Potential benefits in regulating cellular homeostasis in diabetes must be put into the context of the benefits of excellent blood glucose control, which achieves the similar osmotic outcome. The aldose reductase inhibitors share an unfavourable complication profile that makes their use for dubious benefit difficult to defend. It is worth noting that vitamin $\mathrm{C}$ can inhibit aldose reductase and that red blood cell sorbitol levels were normalised after 3 months of vitamin C (100-600 mg) in a study group of type I diabetics. ${ }^{13}$

\section{Abnormalities of rheology and haemostasis}

Increased platelet activation, aggregation, and thromboxane A2 production is well described in diabetics. Other documented haematological abnormalities that would produce a thrombotic tendency in diabetic patients include increased endothelial cell factor VIII production, decreased endothelial cell prostacyclin production, and lower levels of plasminogen activator. There is evidence that leucocytes are less deformable in diabetic subjects and that leucocyte adherence to endothelial surfaces is greater in this condition. These abnormalities should increase the likelihood of capillary occlusion, with formation of microthrombus formation, and this has been demonstrated in cadaver eyes from diabetic subjects which had a high incidence of microthrombus formation. ${ }^{11}$

Aspirin inhibits platelet aggregation. The use of aspirin and other agents aimed at reducing small capillary occlusion from platelet microthrombus formation has been tested in large clinical trials. The DAMAD study 
(Dipyridamole, Aspirin, Microangiopathy of Diabetes) reported in $1989 .^{3}$ This was a large multicentre randomised controlled trial of aspirin alone or in combination with dipyridamole compared to placebo in patients with early diabetic retinopathy. A total of 475 patients with nonproliferative diabetic retinopathy were enrolled. Aspirin dosage was $1 \mathrm{~g} /$ day; dipyridamole dosage was $25 \mathrm{mg} /$ day. Annual microaneurysm counts, based on fluorescein angiography, showed slower annual incrementation of microaneurysms in the treatment groups compared to placebo, which was statistically significant $(P=0.02)$. Treatment effect was uniform across the subset that clinically progressed, but with side effects, primarily gastrointestinal, predominant in the treatment group.

The DAMAD study was designed in 1976 before the advent of low-dose aspirin therapy. Compliance was an issue in this study with $10 \%$ of placebo patients taking aspirin and $20 \%$ of the treatment patients being noncompliant. Despite these problems, the placebo group had a mean increment of 1.44 microaneurysms per year compared to 0.69 microaneurysms in the aspirin group and 0.34 microaneurysms per year in the aspirin/ dipyridamole group. Despite reaching statistical significance, the small change in absolute rates of incrementation of microaneurysm detection meant the authors stopped short of recommending aspirin as a treatment for diabetic retinopathy. However, given other evidence of the benefits of aspirin in macrovascular disease, these results have been taken as evidence supportive of such treatment, ${ }^{3}$ and aspirin is now widely prescribed in diabetic subjects.

The role of aspirin has also been tested in part of the ETDRS Study. ${ }^{4}$ A total of 3711 patients with mild-tosevere nonproliferative or early proliferative diabetic retinopathy were randomised to aspirin (650 mg/day) or placebo. Aspirin did not prevent the development of high-risk proliferative retinopathy and the risk of visual loss, nor did it increase the risk of vitreous haemorrhage.

Thus, the theoretical role for aspirin is confirmed by clinical studies but only in early retinopathy and the absolute clinical improvement achieved with the highdosage aspirin regimen appears too minor to be clinically significant.

Ticlopidine hydrochloride is a therapeutic agent with platelet inhibitory properties. The Ticlopidine, MicroAngiopathy of Diabetes Study (TIMAD) reported in $1990 .{ }^{15}$ This was a multicentre randomised doubleblind placebo controlled trial, in which 435 patients with early diabetic retinopathy were followed and examined by fluorescein angiography annually for 3 years. The average increment in the microaneurysms was significantly higher in the placebo group (1.44) than in the ticlopidine group $(0.48)(P=0.03)$. Subgroup analysis showed the additional benefit among insulin-treated diabetics of whom less developed new vessels in the ticlopidine group $(P=0.056)$. Side effects were predominant in the treatment group, with two patients developing severe neutropaenia, and therefore this agent has not been widely used.

\section{Glycaemic control}

The diabetic control and complications trial (DCCT) was a primary and secondary prevention study that compared tight blood glucose control with routine management of type I diabetes. The main end point was progression of diabetic retinopathy. There was a $76 \%$ reduction in the progression of diabetic retinopathy in the tight control group over the period of 9 years of follow-up. ${ }^{7,16}$ This has highlighted the importance of tight glycaemic control in type I diabetics and has also been used to support the contention that hyperglycaemia is one of the principal aetiologies of end organ damage in diabetes mellitus. ${ }^{17}$ Unfortunately, perfect glycaemic control remains elusive in many patients and there is a cost to the patient of significant hypoglycaemic events in the tight control group. ${ }^{18}$

Following the DCCT, the expectation was generated that a similar improvement in the incidence of diabetic complications would occur in type II diabetes, where tight blood glucose control was exercised. The United Kingdom Prospective Diabetes Study (UKPDS) addressed the issue of tight glycaemic control in type II patients (see Figure 3). ${ }^{6}$ The UKPDS demonstrated a significant but very small absolute reduction in photocoagulation rates $(2.7 \%, P<0.05)$ and progression of retinopathy in intensively treated patients with chlorpropamide, glibenclamide, or insulin compared to those on conventional treatment for 10 years. ${ }^{6,19}$ Of

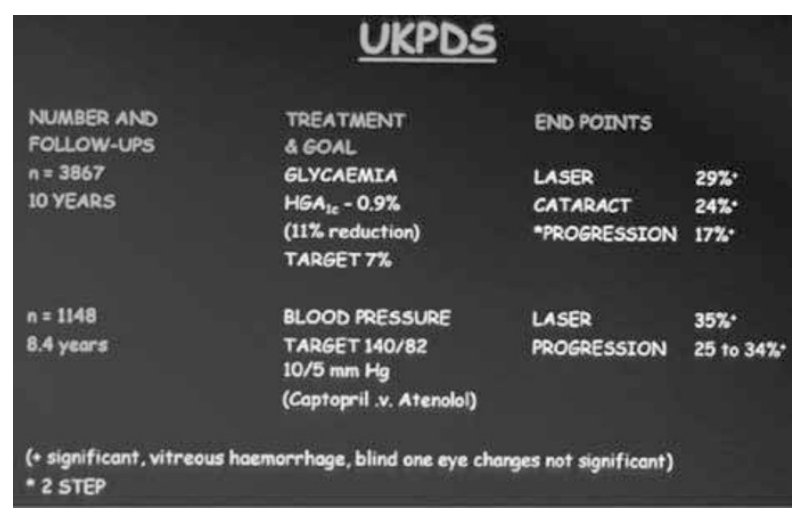

Figure 3 Summary of the UKPDS Study: retinopathy end points. End points are reductions, laser end points were mostly focal laser for maculopathy. 
additional importance was the observation that there is an inexorable rise in glycated haemoglobin levels despite intensive treatment over a 10-year period, thereby demonstrating the difficulty in achieving the glycaemic target of $\mathrm{HbA}_{1} \leqslant 7 \%$. Type I diabetic patients would appear to obtain greater benefit from tight glycaemic control than those with type II diabetes. ${ }^{20}$

\section{Hypertension treatment trials}

Over the past two decades, there have been a large number of landmark cardiovascular treatment trials in diabetic subjects, but the majority have not included microvascular end points, except four studies and in these, confirmation has been provided of the important role of blood pressure control in the treatment of diabetic retinopathy. ${ }^{21}$ There have been a series of reports in the literature showing an association between systemic hypertension and retinal exudation, haemorrhage and maculopathy. ${ }^{22-24}$ Retinal hyperperfusion is believed to be an important initial mode of injury to the retinal microcirculation in diabetes. The retinal vasculature is autoregulated and in normal subjects optimal flow rates are maintained over widely varying levels of systemic perfusion pressure. In diabetic patients autoregulation of the retinal circulation is impaired, leading to increased flow rate, which is proportional to the systemic pressure level. Increased retinal blood flow is found in conditions that worsen diabetic retinopathy such as hypertension, hyperglycaemia, pregnancy, and autonomic neuropathy. In contrast, conditions that reduce retinal blood flow such as carotid artery stenosis and elevated intraocular pressure appear to afford protection from diabetic retinopathy. ${ }^{11}$

The UKPDS published in 1998 was designed as a prospective observational study of 7416 patients with newly diagnosed type II diabetes (diagnostic criteria: fasting plasma glucose concentration $>6 \mathrm{mmol} / 1$ on two separate mornings). The patients were between 25 and 65 years of age and recruitment occurred between 1977 and 1991 at 23 clinical centres in the United Kingdom. A total of 1148 patients with hypertension (diagnostic criteria: $>150 / 80$ receiving treatment; $>160 / 90$ not receiving treatment) were randomised to either tight control of blood pressure with beta-blocker or an angiotensin converting enzyme inhibitor, or to a policy of less tight pressure control (see Figure 3). The aim was to maintain blood pressure $<150 / 85 \mathrm{mmHg}$ in the group randomised to tight blood pressure control, with additional agents being added should this level not be achieved with beta-blocker or ACE inhibition therapy. In the less tight blood pressure control group $(n=390)$ the target pressure was $<180 / 105$ without the use of a betablocker or ACE inhibitor but using the same stepwise addition of other treatments as set out for the tight treatment group. The mean blood pressure during follow-up was $144 / 82 \mathrm{mmHg}$ in the tight control group and $154 / 87 \mathrm{mmHg}$ in the less tight control group $(P<0.0001)$. After 7 years follow-up, for a $10 \mathrm{mmHg}$ reduction in systolic pressure, and $5 \mathrm{~mm}$ reduction in diastolic pressure, there was a $35 \%$ reduction in the proportion of patients with deterioration of retinopathy by two steps on the ETDRS scale $(P=0.004)$. At 9 years, there was $47 \%$ reduction in the risk of a decrease in vision by three or more lines in both eyes measured with an ETDRS chart $(P=0.004)$. Photocoagulation was reduced by $35 \%$ in the tight control group $(P=0.025)$. Most of the reduction in photocoagulation was from a reduced requirement for macular photocoagulation, which accounted for $78 \%$ of laser treatments performed. ${ }^{8}$

Other studies of hypertension in type II diabetes have confirmed the benefit of blood pressure control. The Steno II study in type II diabetes with microalbuminuria investigated the intensive control of hypertension, hyperglycaemia and hypercholesterolaemia, with 73 patients receiving intensive treatment of these multiple risk factors and 76 patients being randomised to standard treatment. ACE inhibitors were used irrespective of blood pressure in the intensively treated group but restricted to hypertensive patients in the standard treatment group. At 4 years of follow-up, one patient in the intensive treatment group had become blind in one eye, compared to seven patients in the standard treatment group $(P=0.03$ ) (see Figure 4$){ }^{17,25}$

The EUCLID randomised blind controlled study, which reported in 1998, investigated the use of an ACE inhibitor (lisinopril) in patients with type I diabetes who were predominantly normotensive. After 2 years of follow-up, the ACE inhibitor group had statistically significant reduction of $50 \%$ in the progression of retinopathy by at least 1 Eurodiab level $(P=0.02)$ compared to placebo (see Figure 5). ${ }^{9,26}$ The findings of

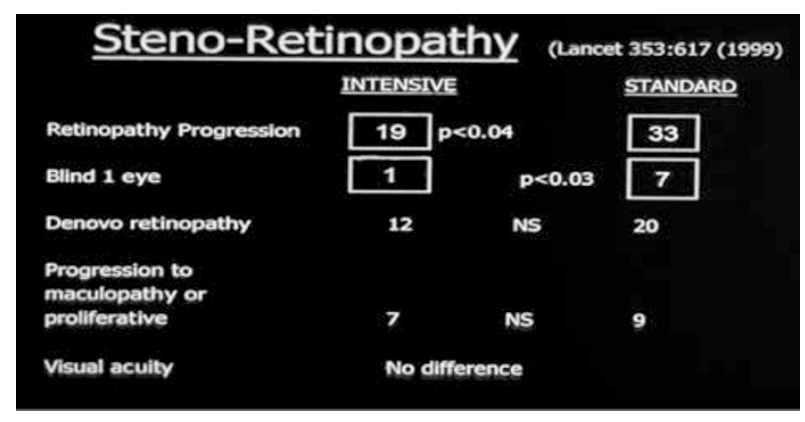

Figure 4 Steno 2 trial: principal retinopathy results. Intensive treatment was with ACE inhibitors, statins, aspirin, and tight glycaemic control. Mean follow-up 4 years. 


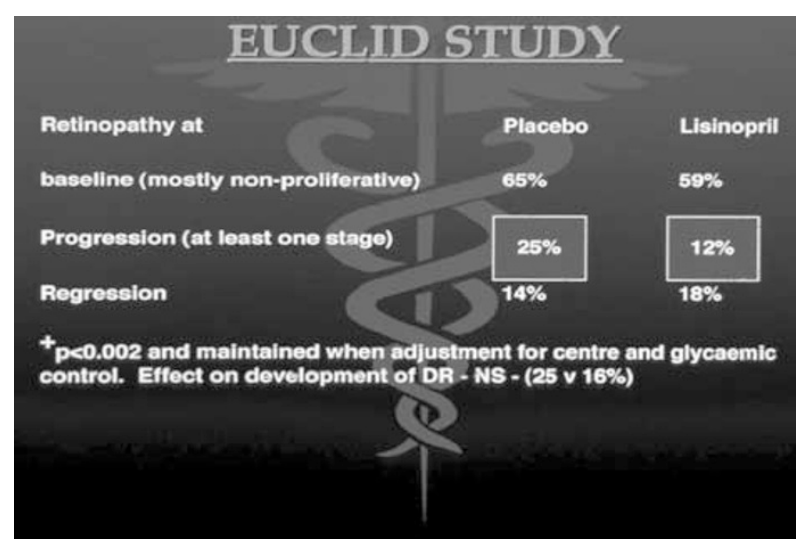

Figure 5 Principal retinopathy results of EUCLID study. DR, diabetic retinopathy, NS, nonsignificant. Trial in 325 type I diabetics, results at 2 years.

this study were weakened owing to differences in initial and final glycated haemoglobin levels favouring lisinopril.

Do ACE inhibitors offer greater protection to the retina than other forms of antihypertensive treatments in diabetic patients? The UKPDS, which demonstrated outstanding benefit of tight control of blood pressure in hypertensive type II diabetics, failed to show any benefit of ACE inhibitor over beta-blocker. ${ }^{8}$ The contradiction between the UKPDS and EUCLID study findings may be explained by the insufficient statistical power to assess the effects of the different third-line antihypertensive agents used in the UKPDS study. However, The Heart Outcomes Protection Evaluation Study (HOPE Study), which was a multicentre study of 9500 patients over the age of 55 years, known to be at high risk of

cardiovascular disease, ${ }^{10}$ has supported the specific effect of ACE inhibition. The study was a double-masked randomised multicentre comparison of Ramipril vs placebo in patients at high cardiovascular risk. Of the 9500 patients, 3500 were diabetic. The diabetic subgroup had to either have known cardiovascular disease or one other risk factor including cholesterol $>5.2 \mathrm{mmol} / 1$ or blood pressure $>140 / 90 \mathrm{mmHg}$, cigarette smoking or microalbuminurea. At entry patients could be taking any medication except ACE inhibitors and the planned follow-up time was 5-6 years. The study was in fact stopped at 4 years, as a clear benefit in favour of Ramipril over placebo had become manifest. There was an overall reduction in mortality of over $20 \%$ with ACE inhibitor therapy. The study was not designed to show an effect on diabetic retinopathy; however, there was a trend to reduction in laser treatment for diabetic retinopathy in the Ramipril group.

Although the benefit of ACE inhibitors in preventing diabetic nephropathy and reducing mortality from

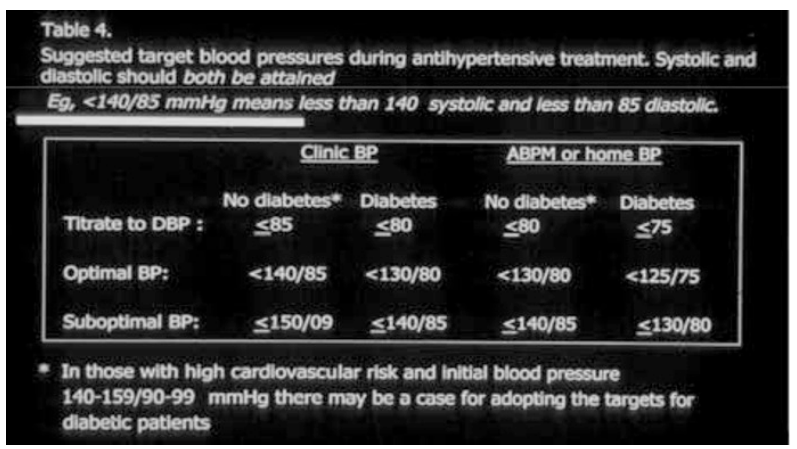

Figure 6 Blood pressure targets defined by the British Hypertension Society (1999). Adapted from J Human Hypertens 13: 569-592 (1999).

cardiovascular disease in diabetic patients is proven, a benefit of ACE inhibitors in delaying the ocular complications of diabetes is likely but less well confirmed. Tight control of blood pressure (see Figure 6) does protect the retina from visual loss, particularly maculopathy in type II diabetics. If blood pressure reduction can be achieved with ACE inhibition, then this provides additional nonocular benefits. ${ }^{27}$

Angiotensin II receptor blockers are a new class of drug used in the treatment of hypertension. Losartan was the first angiotensin II blocker in clinical use, followed by a further five agents. Use of angiotensin II blockers is associated with lower rates of the troublesome cough that is a prominent side effect of ACE inhibitors, because the bradykinin system is unaffected by angiotensin II blockade. ${ }^{28}$ A potential benefit of specific angiotensin II receptor blockade is an antiangiogenic effect that these agents have been shown to have in vitro. ${ }^{29,30}$ Although there is no trial data of angiotensin II blockers in patients with retinopathy, these agents are looking very promising with beneficial effects being demonstrated in large randomised trials in patients with diabetic nephropathy (losartan on cardiovascular and renal outcomes, irbesartan on development of diabetic nephropathy (see Figure 7$)^{31-34}$ ). In addition, the recent LIFE study has confirmed that losartan prevented more cardiovascular morbidity and death than atenolol, and was better tolerated. ${ }^{35}$ While we await a head to head comparison of ACE inhibitors and angiotensin II receptor blockers, one large controlled randomised study of the angiotensin II blockade (DIRECT study) in patients with diabetic retinopathy is ongoing.

\section{Cholesterol-lowering treatments}

Several reports have established the association of diabetic exudative maculopathy with hypercholesterolaemia and combined 


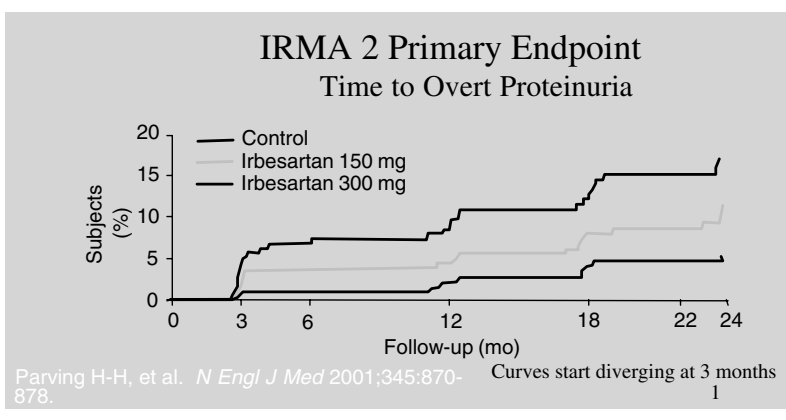

Figure 7 Effect of irbersartan on the development of diabetic nephropathy in type II diabetes (IRMA). Trial of 590 hypertensive type II diabetic subjects, double-blind randomised trial comparing irbesartan treatment to placebo, over 2 years. Primary end point $=$ persistent albuminuria $>200 \mu \mathrm{g} / \mathrm{min}^{32}$

hyperlipidaemia. ${ }^{22,36,37}$ The predictive value of exudative retinopathy for mortality has also been described.

Cardiovascular treatment trials have proven that inhibitors of 3-hydroxy-3-methylglutaryl coenzyme A (HMG-CoA) reductase (statins) significantly reduce cardiovascular-related morbidity and mortality in patients with and without coronary artery disease. ${ }^{17}$

Statins lower plasma LDL-cholesterol concentrations by inhibiting hepatic cholesterol synthesis. It has been established that improvements in cardiovascular outcomes occur years before any measurable improvements in plaque dimensions, and do not relate to the initial level of serum cholesterol levels. Statins alter macrophage cholesterol synthesis, and inhibit plateletderived growth factor (PDGF) induced DNA synthesis, improve blood flow by an effect on vascular endothelium, modulate platelet thrombus formation by reducing the cholesterol content of the membranes of both erythrocytes and platelets, and may lower blood pressure and homocysteine levels. ${ }^{38}$

While the use of statins is guided by the cardiovascular treatment trials, there are studies showing an association between retinal exudation and increased baseline cholesterol levels, ${ }^{36}$ such that increasing cholesterol levels are now an established risk factor for diabetic retinopathy. Early small intervention studies showed reversal or stabilisation of macular exudation with cholesterol-lowering treatment but with no change in visual acuity in patients with advanced diabetic macular disease, and hence this therapy was not further investigated for some years. A more recent small uncontrolled study of pravastatin in diabetic patients with exudative maculopathy reopened this therepeutic approach as it demonstrated reduction of exudation and improvement of background diabetic retinopathy in all six subjects over 1 year. All subjects had hyperlipidaemia and three received supplementary cholestyramine because of failure to achieve the target serum cholesterol levels. ${ }^{39}$

The mechanisms by which cholesterol-lowering agents might improve exudative diabetic retinopathy is not established. Basic research has suggested that oxidised LDL cholesterol is toxic to retinal endothelial cells, and that statins may improve endothelial function, platelet aggregation, and reduce growth factor-induced DNA synthesis. It also opens the possibility that the ocular benefits of these agents may not be restricted to only impeding exudate formation. Further investigation is underway with two large randomised studies assessing diabetic retinopathy with detailed photography before and during statin therapy (FIELD and ASPEN studies). The ASPEN study will report on fundal photographs of 511 patients with diabetic retinopathy who have been randomised to either atorvastatin $10 \mathrm{mg}$ or placebo. This study is due to complete in 2003.

\section{Endocrine and paracrine growth factors}

Prior to the publication of the ETDRS and the widespread availability of argon laser photocoagulation, pituitary ablation was a treatment option for proliferative diabetic retinopathy. This treatment was founded on a case report of a young woman with proliferative diabetic retinopathy whose retinopathy was observed to improve after she suffered postpartum infarction of her pituitary gland. ${ }^{40}$ Pituitary ablation became an established treatment in the 1970s and a number of uncontrolled series were published. Improvement in the degree of proliferative retinopathy was found to correlate with the postablation level of growth hormone, determined on an insulin stress test, and correlated with decreasing levels of peak serum growth hormone production. ${ }^{41}$ However, half of the patients who achieved maximum postablation reductions of growth hormone production demonstrated no change or even worsening of diabetic proliferative retinopathy. ${ }^{41}$

Subsequently, research into the role of growth factors in angiogenesis has focused on the release of the major angiogenic growth factors produced from retinal tissue in response to retinal ischaemia. Identified local retinal angiogenic growth factors are vascular endothelial growth factor (VEGF), insulin-like growth factor 1 (IGF1), and PDGF, with growth factors derived from ischaemic retinal tissue being more important in the causation of proliferative diabetic retinopathy than pituitary-derived growth factors. ${ }^{42}$

A natural inhibitor of the action of growth hormone and IGF1 is somatostatin which could have therepeutic potential. Somatostatin is produced by tissues of the neuroendocrine and gastrointestinal systems and has broad inhibitory actions which also include action 
against TSH, prolactin, glucagon, insulin, and pancreatic exocrine function. Somatostatin is known to have an inhibitory effect on tumour growth that was found to be because of a direct antiproliferative effect in addition to inhibition of growth factors. Somatostatin activates intracellular protein tyrosine phosphatases that deactivate growth factor receptors and in turn inactivate cellular responses promoted by growth factors.

Octreotide is a somatostatin analogue that has a halflife of $90 \mathrm{~min}$ and an 8-h duration of action and is administered by subcutaneous injection. Octreotide is 20 times more potent than somatostatin in inhibiting growth hormone secretion ${ }^{38}$ and is well documented in the treatment of acromegaly. Several small clinical trials have assessed its usefulness in treatment of proliferative diabetic retinopathy.

There have been several trials of somatostatin analogues for the prevention of the complications of diabetic retinopathy, with conflicting results. ${ }^{43-47}$ The side-effect profile is significant as demonstrated in the study by Kirkegarrd. Patients randomised to Octreotide received the treatment by continuous subcutaneous infusion for 12 months, three patients discontinued Octreotide because of gastrointestinal effects. ${ }^{47}$ More recently, results of a pilot study were published which examined the ability of Octreotide to retard the progression of diabetic retinopathy and delay the need for pan retinal photocoagulation in patients with advanced stages of retinal disease. A total of 22 patients with severe nonproliferative diabetic retinopathy or early nonhigh-risk proliferative diabetic retinopathy were randomly assigned to conventional diabetes management $(n=12)$ or additional treatment with maximally tolerated doses of Octreotide by subcutaneous administration ( $n=11$ patients). The dosing regimen was Octreotide four times a day or by continuous subcutaneous infusion. Doses were individually adjusted to the highest tolerated levels of Octreotide. This was determined to have occurred when the IGF1 level was lowered to the level equivalent to patients with hypopituitarism, or there was intervention of drugrelated adverse effects, specifically diarrhoea. Ocular examination was carried out 3 monthly for 15 months in each participant or until a laser surgery end point was reached. All patients in the treatment group received thyroxin treatment to ensure clinical euthyroid status throughout the trial. The results of this approach were as follows:

One patient in the Octreotide group required pan retinal photocoagulation (PRP) whereas in the conventionally managed group of patients, nine eyes required PRP. Kaplan-Meier survival curves demonstrated a significant difference between the time profiles to PRP between the groups. However, the proportion of eyes that required PRP in either group was not significantly different at the 0.05 level. ${ }^{48}$ The authors also speculated that thyroid supplementation in this study could have potentiated the action of Octreotide at delaying proliferative diabetic retinopathy. The mechanism of action of somatostatin is unclear but it may be because of a direct antiangiogenic effect as well as inhibition of growth hormone and IGF1 secretion. There has been one report documenting the efficacy of Octreotide in treating macular oedema that was refractory to anti-inflammatory drugs and acetazolamide. ${ }^{49}$

A further pilot study has evaluated the usefulness of Octreotide in the group of patients who had persisting proliferative diabetic retinopathy after extensive argon laser photocoagulation. Using a dosing regimen of three subcutaneous injections of $100 \mu \mathrm{g} /$ day, there were nine (ie 18 eyes) patients who received Octreotide and nine controls. Neovascularisation decreased in 14 of 18 treated eyes and there were no further vitreous haemorrhages (no vitrectomy requirement) or decrease in visual acuity over the 3-year follow-up period. However, in the control group, vitreous haemorrhage occurred in eight of 18 eyes and three eyes required vitrectomy. ${ }^{50}$

The results of the latter two preliminary studies of Octreotide in proliferative diabetic retinopathy are promising. With the advent of longer acting somatostatin analogues, for example, lanreotide and sandostatin LAR, the feasibility of this therepeutic is much enhanced, such that a large intervention study using one of these longacting preparations is well underway, and due to report in the next 2 years. More recently, growth hormone receptor antagonists (GHRA) have been developed by genetic engineering. One such is pegvisomant which is projected to have a reversible binding to the human growth hormone receptor with an affinity substantially exceeding that of human growth hormone, which leads to reductions in circulating IGF1 levels. ${ }^{51}$ Trials of this therapy are ongoing in acromegaly, and one small uncontrolled trial has been performed in patients with proliferative diabetic retinopathy $(n=25)$, using a daily subcutaneous dosage of $20 \mathrm{mg}$ for 12 weeks. ${ }^{51}$ While regression of retinopathy was not observed in any patient, 16 patients did not demonstrate progression of retinopathy, and in nine patients the retinopathy grade deteriorated. This study has therefore not supported an obvious effect of this therapy, but has confirmed that this therapy has a better side-effect profile than octreotide, such that controlled studies should be performed.

\section{Vascular endothelial growth factor}

Vascular permeability is dramatically altered by the local action of growth factors, hormones, and intraocular 
inflammatory mediators on the retinal microvasculature. VEGF is a glycopeptide which binds to specific tyrosine kinase receptors (FLT1 and KDR) on vascular endothelial cells. VEGF, formerly known as vascular permeability factor (VPF), has been shown to cause profound increase in the permeability of normal retinal vessels in experimental animals, ${ }^{52}$ probably by an effect on intercellular adhesion molecules (ICAM), and is also a potent stimulus for neovascularisation.

Growth factors are important for maintaining and promoting the normal function of retinal vascular and paravascular cells. VEGF has an important role in vascular cell survival and the maintenance of the fenestrations of the choriocapillaris. VEGF is expressed by vascular and neuroglial cells and is recognised as a key growth factor involved in the genesis of exudative and proliferative diabetic retinopathy. ${ }^{53}$

The data relating VEGF in the pathogenesis of diabetic retinopathy are compelling. Hyperglycaemia increases the production of VEGF in the retina, and this hyperglycaemia-induced VEGF gene expression in human endothelial cells has been shown to be mediated by a protein kinase C (PKC)-dependant mechanism. ${ }^{54}$

VEGF expression is triggered by hypoxia, and in proliferative diabetic retinopathy it is expressed in the vitreous and in the vicinity of preretinal new vessels. Levels of VEGF decline following laser photocoagulation. Novel anti-VEGF proteins, administered by intravitreal injection, have been studied in human subjects with exudative macular degeneration but not in patients with diabetic retinopathy. ${ }^{55}$ The importance to diabetic retinopathy of the PKC pathway and VEGF production has been reported in animal models, with randomised controlled studies being currently conducted.

\section{Protein kinase $\mathrm{C}$ enzyme}

PKC is a family of at least 12 isoenzymes. The kinase enzymes add phosphate groups to proteins and thereby activate key metabolic enzymes. They are ubiquitous enzymes and can be subdivided into those that phosphorylate proteins at tyrosine residues (tyrosine kinases) and those that phosphorylate serine and threonine sites (serine threonine kinases). PKC is one of two major serine threonine kinases, the other being cyclic AMP protein kinase. PKC phosphorylates a large number of intracelluar substrates including the insulin receptor and some key enzymes involved in glucose metabolism. ${ }^{56}$

The VEGF receptor signal transduction in the retina is mediated via the PKC beta I and II isoforms. Independent activation of these can alter vascular functions in diabetics including permeability, coagulation, and blood flow. Diacyglycerol (DAG), which is produced during glycolysis and is elevated in diabetes, is the most potent known activator of PKC, and hence leads to an increase in VEGF production (see Figure 8). There are a growing number of interactions of PKC, which possibly suggests a unique role for this enzyme in diabetic pathophysiology. PKC beta isoform activation has been shown to inhibit the sodium potassium ATPase pump, and is known to upregulate genes coding for basement membrane proteins such as fibronectin and type IV collagen (see Figure 9). ${ }^{57,58}$

Early inhibitors of PKC (H7 and Staurosporine) blocked all PKC isoforms (A, B, and C) and consequently had limited clinical usefulness. Vitamin $\mathrm{E}$ is known to decrease glucose-induced PKC activation by promoting the clearance of DAG via an effect on DAG kinase but the effect is not strong. ${ }^{59}$ The recent discovery of LY333531, a specific inhibitor of the PKC beta isoforms, that can be taken orally, has opened a new research chapter. In early experimental studies, this compound has been shown to reverse diabetes-induced abnormalities of retinal blood

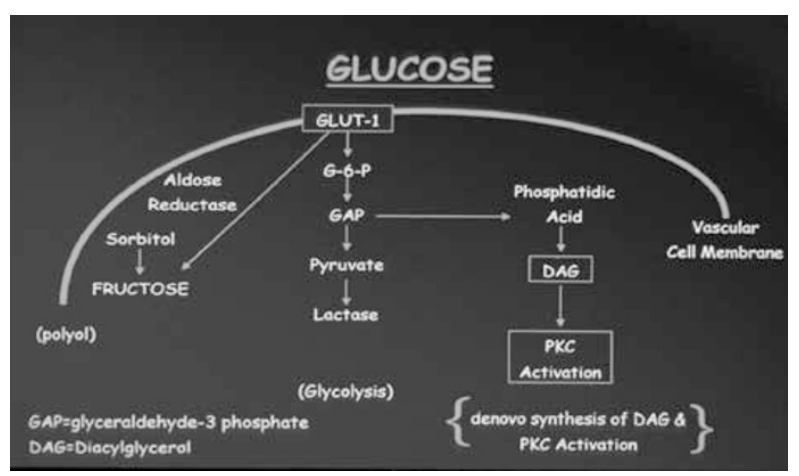

Figure 8 Glucose conversion to protein kinase C activation. Glucose activates the GLUT-1 receptor and in excess leads to increased diacylglycerol formation with activation of the protein kinase C system.

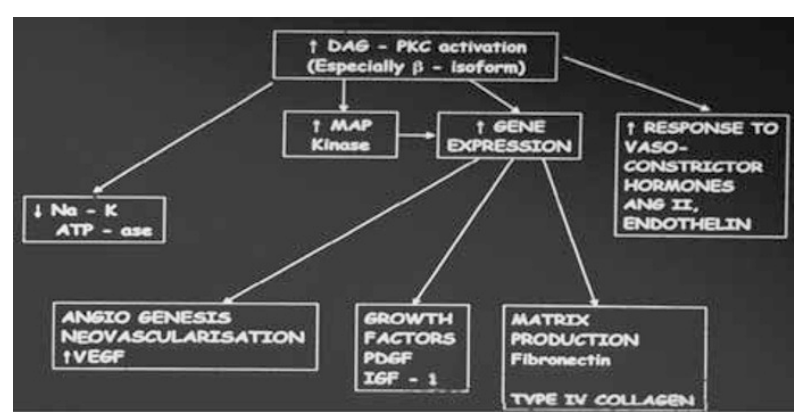

Figure 9 Results of activation of the PKC beta isoform pathway. DAG, diacylglycerol; Ang 11, angiotensin 11; PDGF, platelet-derived growth factor; IGF-1, insulin growth factor 1; PKC, protein kinase C; VEGF, vascular endothelial growth factor. 
flow, glomerular filtration rate, albuminuria, ${ }^{60}$ and suppress retinal permeability induced by $\mathrm{VEGF}^{61}$ in experimental animals (see Figure 10). The antiangiogenic potential has also been shown, with inhibition of PKC II beta isoform activity resulting in reduced proliferation of endothelial cells induced by VEGF, in a pig model of laser-induced retinal vein occlusion with measurement of iris rubeosis. ${ }^{62}$ This specific inhibitor of the PKC system offers the prospect of the first oral specific medication to reduce diabetic microvascular disease and specifically diabetic retinopathy. A large randomised clinical trial programme is currently being performed in diabetic microvascular complications in man, with pivotal studies in patients with macula oedema and preproliferative diabetic retinopathy. Whether this exciting approach will alter the natural history of diabetic retinopathy or be an adjunct to laser therapy, will be answered by the trials. The trial results are expected to be available in 2003.

\section{Protein glycation}

The nonenzymatic reaction of reducing sugars with the amino groups of peptides and proteins, which results in the formation of complex brown pigments and protein crosslinks, is referred to as protein glycation. Under conditions of hyperglycaemia, glucose binds nonenzymatically to proteins to form ketoamines which undergo a further series of nonenzymatic reactions, ultimately resulting in the formation of advanced glycosylation end products or AGEs. ${ }^{63}$ The formation of glycosylated haemoglobin (HbA1) is an example of the formation of a reversible intermediate by the process of protein glycation. The plasma concentration of $\mathrm{HbA} 1$ defines equilibrium between glycated and nonglycated proteins and is an established correlate of glycaemic control over the preceding 28-day period in diabetic patients. $^{63}$

AGEs, however, are resistant to degradation and may accumulate throughout life, particularly in connective

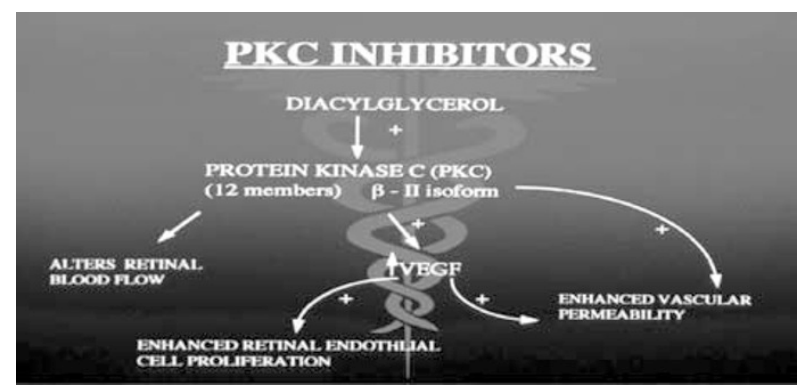

Figure 10 Summary of protein kinase $C$ activation on the retina. VEGF, vascular endothelial growth factor. New PKC beta isoform inhibitor blocks the action of this enzyme. tissues, including the vasculature, in which protein turnover is low. ${ }^{63}$ AGE modifications have serious consequences for macromolecular function, especially in the case of DNA, ${ }^{64}$ important structural proteins. ${ }^{65}$ enzymes, ${ }^{66}$ and growth factors. ${ }^{67}$

Furthermore, specific AGE receptors are found on a variety of cell types. Complex receptor systems exist to remove senescent, glycation-modified molecules and limit their deleterious effects. AGEs can also initiate a wide range of abnormal responses in cells and tissues such as inappropriate expression of growth factors, alterations in growth dynamics, accumulation of extracellular matrix, promotion of vasoregulatory dysfunction, and initiation of apoptotic cell death pathways. ${ }^{67}$

In a comprehensive review, Ulrich and Cerami ${ }^{63}$ outline the role protein glycation may have in the development of the complications of diabetes, and describe the pharmacological approaches to inhibiting glycation and reversing its effects. There are several published reports on the ability of aminoguanidine to inhibit AGE formation in vivo. ${ }^{68-77} \mathrm{~A}$ recent report showed the ability of aminoguanidine to prevent the development of diabetic retinopathy in diabetic dogs followed for 5 years. ${ }^{78}$ Optimism for the clinical use of aminoguanidine has been tempered by the gradual realisation that aminoguanidine also inhibits a range of other important pathways, most notably generation of nitric oxide by eNOS, which may increase nonspecific and unwanted side effects of the drug. ${ }^{67}$ Nevertheless, aminoguanidine and/or other related AGE inhibitors may eventually find a place in the management of diabetics or in individuals at risk of age-related sequelae. ${ }^{67}$

Recently, compounds have also been developed that are capable of breaking pre-established AGE proteinprotein crosslinks. ${ }^{79-81}$ Two crosslink breaker compounds are undergoing evaluation: 3-phenacylthiazolium bromide (PTB) and 4.5-dimethyl-3-phenacylthiazolium chloride (DPTC) (ALT-711). These compounds, which share a common thiazolium structure, have the ability to reduce tissue content of AGEs in experimental diabetes, ${ }^{80}$ ameliorate age-related myocardial stiffness, ${ }^{81}$ and reverse hyperglycaemia-related arterial distensibility. ${ }^{73,82}$

\section{Oxidation}

Free radicals are unstable chemical derivatives of oxygen that carry unpaired electrons and are the by-products of metabolism, especially mitochondrial oxidative phosphorylation. Increased oxidative stress, giving rise to free radical-mediated damage to proteins, nucleic acid, and lipids, is a plausible mechanism of injury in many disease states including diabetes. ${ }^{83}$ 
There are reports of increased lipid peroxidation in diabetes, particularly in relation to microangiopathy. ${ }^{84}$ Samples from patients undergoing vitrectomy for proliferative diabetic retinopathy showed increased free radical formation and decreased antioxidant activity in the human vitreous body compared to vitrectomy samples from eyes with nonproliferative indications. ${ }^{85} \mathrm{~A}$ total of 13 type II diabetics with retinopathy given alpha tocopherol nicotinate had reduction in blood viscosity documented at 3 months, attributed to reduction of lipid peroxidation stress on erythrocyte cell walls by the authors. ${ }^{86}$ Blood flow abnormalities in the retinal circulation of experimental rats with induced diabetes were prevented by intraperitoneal doses of d-alpha tocopherol. ${ }^{87}$

Diabetic patients have been found to have lower concentrations of reduced glutathione, vitamin $\mathrm{C}$, and vitamin $\mathrm{E}$, but not all studies have shown similar reductions. ${ }^{88}$ It has been postulated that the reduction in antioxidant reserve in diabetic patients may be because of competition for NADPH, from the sorbitol pathway. ${ }^{13}$

While this appeared a promising mechanism to manipulate with well-tolerated vitamin supplements, the results of clinical studies of antioxidant supplementation have been, by and large, disappointing. The largest randomised controlled study to investigate this approach was the HOPE study. In this, 3577 diabetics over the age of 55 years were randomised to receive either $400 \mathrm{IU}$ of vitamin E daily or placebo, for 4.5 years. Although, as previously mentioned, outstanding reductions in the rates of the primary cardiovascular end points was witnessed in participants randomised to Ramipril in this study, with a trend towards less laser interventions for retinopathy, in contrast, no beneficial effect was observed from the usage of vitamin E supplementation in this study. ${ }^{10}$

\section{Conclusions}

The quest for specific medical therapies to influence the course of diabetic retinopathy has been fraught (hence 'graveyard' in the title of this article), with apparently promising agents (aldose reductase inhibitors, aspirin, and vitamin $\mathrm{E} / \mathrm{C}$ ) being ineffective, and others too toxic for use in man (aminoguanidine).

The emphasis in current diabetes practice is on screening, delivery of laser treatment and achievement of recommended targets for glycaemic control, blood pressure, and lipids, with widespread use of ACE inhibitors, statins, and aspirin therapies, in addition to hypoglycaemic agents. ${ }^{89,90}$

The recent realisation of the importance of growth factors (eg VEGF, IGF1) in the development of diabetic retinopathy has led to a new potential chapter in targetting treatments. The discovery of a PKC beta inhibitor, reducing the effects of VEGF, has led to an exciting clinical development programme, due to report the results of the initial controlled studies (preproliferative and diabetic maculopathy) in the near future. This approach, in addition to growth hormone antagonists by long-acting analogues of octreotide, growth hormone receptor blockers, new glycation inhibitors, and angiotensin receptor antagonists, and statins, may well herald a single or a multiple of new specific treatments for diabetic retinopathy, which will be most welcome to patients and their carers.

\section{References}

1 Anonymous. Preliminary report on effects of photocoagulation therapy. The Diabetic Retinopathy Study Research Group. Am J Ophthalmol 1976; 81(4): 383-396.

2 Anonymous. Photocoagulation for diabetic macular edema. Early Treatment Diabetic Retinopathy Study report number 1. Early Treatment Diabetic Retinopathy Study research group. Arch Ophthalmol 1985; 103(12): 1796-1806.

3 The DAMAD Study Group. Effect of aspirin alone and aspirin plus dipyridamole in early diabetic retinopathy. A multicenter randomized controlled clinical trial. The DAMAD Study Group. Diabetes 1989; 38(4): 491-498.

4 Early Treatment Diabetic Retinopathy Study Research Group. Effects of aspirin treatment on diabetic retinopathy. ETDRS report number 8. Early Treatment Diabetic Retinopathy Study Research Group. Ophthalmology 1991; 98(Suppl 5): 757-765.

5 Sorbinil Retinopathy Trial Research Group. A randomized trial of sorbinil, an aldose reductase inhibitor, in diabetic retinopathy. Sorbinil Retinopathy Trial Research Group. Arch Ophthalmol 1990; 108(9): 1234-1244.

6 Stratton IM, Adler AI, Neil HA, Mathews DR, Manley SE, Cull CA, et al. Association of glycaemia with macrovascular and microvascular complications of type 2 diabetes (UKPDS 35): prospective observational study. BMJ 2000; 321(7258): 405-412.

7 Diabetes Control and Complications Trial Research Group. Progression of retinopathy with intensive versus conventional treatment in the Diabetes Control and Complications Trial. Diabetes Control and Complications Trial Research Group. Ophthalmology 1995; 102(4): 647-661.

8 Adler AI et al. Association of systolic blood pressure with macrovascular and microvascular complications of type 2 diabetes (UKPDS 36): prospective observational study. BMJ 2000; 321(7258): 412-419.

9 The EUCLID Study Group. Randomised placebo-controlled trial of lisinopril in normotensive patients with insulindependent diabetes and normoalbuminuria or microalbuminuria. The EUCLID Study Group. Lancet 1997; 349(9068): 1787-1792.

10 Heart Outcomes Prevention Evaluation Study Investigators. Effects of ramipril on cardiovascular and microvascular outcomes in people with diabetes mellitus: results of the HOPE study and MICRO-HOPE substudy. Heart Outcomes Prevention Evaluation Study Investigators. Lancet 2000; 355(9200): 253-259. 
11 Archer DB. Bowman Lecture 1998. Diabetic retinopathy: some cellular, molecular and therapeutic considerations. Eye 1999; 13(Part 4): 497-523.

12 Van Heyningen R. Formation of polyols by the lens of the rat with sugar cataract. Nature 1959; 184: 194-195.

13 Crabbe MJ, Goode D. Aldose reductase: a window to the treatment of diabetic complications? Prog Retin Eye Res 1998; 17(3): 313-383.

14 van Gerven JM et al. Effects of aldose reductase inhibition with tolrestat on diabetic retinopathy in a six months double blind trial. Doc Ophthalmol 1994; 87(4): 355-365.

15 The TIMAD Study Group. Ticlopidine treatment reduces the progression of nonproliferative diabetic retinopathy. The TIMAD Study Group. Arch Ophthalmol 1990; 108(11): 1577-1583.

16 The relationship of glycemic exposure (HbA1c) to the risk of development and progression of retinopathy in the diabetes control and complications trial. Diabetes 1995; 44(8): 968-983.

17 Dodson PM, Kritzinger EE. Medical cardiovascular treatment trials: relevant to medical ophthalmology in 1997? Eye 1997; 11(Part 1): 3-11.

18 The DCCT Research Group. Epidemiology of severe hypoglycemia in the diabetes control and complications trial. The DCCT Research Group. Am J Med 1991; 90(4): 450-459.

19 Gray A et al. Cost effectiveness of an intensive blood glucose control policy in patients with type 2 diabetes: economic analysis alongside randomised controlled trial (UKPDS 41). United Kingdom Prospective Diabetes Study Group. BMJ 2000; 320(7246): 1373-1378.

20 McCormack J, Greenhalgh T. Seeing what you want to see in randomised controlled trials: versions and perversions of UKPDS data. United Kingdom prospective diabetes study. BMJ 2000; 320(7251): 1720-1723.

21 Gillow JT, Gibson JM, Dodson PM. Hypertension and diabetic retinopathy - what's the story? Br J Ophthalmol 1999; 83(9): 1083-1087.

22 Dodson PM, Gibson JM. Long-term follow-up of and underlying medical conditions in patients with diabetic exudative maculopathy. Eye 1991; 5(Part 6): 699-703.

23 Klein $\mathrm{R}$ et al. Is blood pressure a predictor of the incidence or progression of diabetic retinopathy? Arch Intern Med 1989; 149(11): 2427-2432.

24 Klein $\mathrm{R}$ et al. The Wisconsin Epidemiologic Study of Diabetic Retinopathy: XVII. The 14-year incidence and progression of diabetic retinopathy and associated risk factors in type 1 diabetes. Ophthalmology 1998; 105(10): 1801-1815.

25 Gaede $\mathrm{P}$ et al. Intensified multifactorial intervention in patients with type 2 diabetes mellitus and microalbuminuria: the Steno type 2 randomised study. Lancet 1999; 353(9153): 617-622.

26 Chaturvedi $\mathrm{N}$ et al. Effect of lisinopril on progression of retinopathy in normotensive people with type 1 diabetes. The EUCLID Study Group. EURODIAB Controlled Trial of Lisinopril in Insulin-Dependent Diabetes Mellitus. Lancet 1998; 351(9095): 28-31.

27 Barnett AH. The Heart Outcomes Protection Evaluation (HOPE) study: relevance to ophthalmological practice? Eye 2000; 14(Part 1): 1-2.

28 McInnes GT. Clinical potential: angiotensin converting enzyme inhibitor or angiotensin II antagonist? J Hypertens Suppl 2001; 19(Suppl 1): S61-S67.
29 Gilbert RE et al. Angiotensin converting enzyme inhibition reduces retinal overexpression of vascular endothelial growth factor and hyperpermeability in experimental diabetes. Diabetologia 2000; 43(11): 1360-1367.

30 Unger T, Azizi M, Belz GG. Blocking the tissue reninangiotensin system: the future cornerstone of therapy. J Hum Hypertens 2000; 14(Suppl 2): S23-S31.

31 Lewis EJ, Hunsicker LG, Clarke WR et al. Renoprotective effect of the angiotensin-receptor antagonist irbesartan in patients with nephropathy due to type 2 diabetes. $N$ Engl $J$ Med 2001; 345: 851-860.

32 Parving HH, Lehnert $\mathrm{H}$, Brochner-Mortensen $\mathrm{J}$ et al. The effect of irbesartan on the development of diabetic nephropathy in patients with type 2 diabetes. $N$ Engl J Med 2001; 345: 870-878

33 Brenner BM, Cooper ME, De Zeeuw D et al. Effects of losartan on renal and cardiovascular outcomes in patients with type 2 diabetes and nephropathy. N Engl J Med 2001; 345: 861-869.

34 Hostetter TH. Prevention of end-stage renal disease due to type 2 diabetes. N Engl J Med 2001; 345: 910-913.

35 Dahlof B, Devereux RB, Kleldsen SE et al. Cardivascular morbidity and mortality in the Losartan Intervention for Endpoint reduction in hypertension study (LIFE): a randomised trial against atenolol. Lancet 2002; 359: 995-1003.

36 Chew EY et al. Association of elevated serum lipid levels with retinal hard exudate in diabetic retinopathy. Early Treatment Diabetic Retinopathy Study (ETDRS) Report 22 Arch Ophthalmol 1996; 114(9): 1079-1084.

37 Klein BE et al. The Wisconsin Epidemiologic Study of Diabetic Retinopathy. XIII. Relationship of serum cholesterol to retinopathy and hard exudate. Ophthalmology 1991; 98(8): 1261-1265.

38 Vaughan CJ, Murphy MB, Buckley BM. Statins do more than just lower cholesterol. Lancet 1996; 348(9034): 1079-1082.

39 Gordon B et al. The effects of lipid lowering on diabetic retinopathy. Am J Ophthalmol 1991; 112(4): 385-391.

40 Poulsen J. Recovery from retinopathy in a case of diabetes with Simmond's disease. Diabetes 1953; 2: 7-12.

41 Wright $\mathrm{AD}$ et al. Serum growth hormone levels and the response of diabetic retinopathy to pituitary ablation. $B M$ ) 1969; 2(653): 346-348

42 Forrester JV, Olson J, Pascal M, Robertson M, Muckersie E. Growth factors and diabetic retinopathy. Diabetes Rev Int 1997; 6(1): 9-11.

43 Grant MB, Caballero S, Millard WJ. Inhibition of IGF-1 and b-FGF stimulated growth of human retinal endothelial cells by the somatostatin analogue, octreotide: a potential treatment for ocular neovascularization. Regul Pept 1993; 48(1-2): 267-278

44 McCombe $\mathrm{M}$ et al. Effect of a long-acting somatostatin analogue (BIM23014) on proliferative diabetic retinopathy: a pilot study. Eye 1991; 5(Part 5): 569-575.

45 Mallet B et al. Stabilization of severe proliferative diabetic retinopathy by long-term treatment with SMS 201-995. Diabetes Metab 1992; 18(6): 438-444.

46 Shumak SL et al. Growth hormone suppression and nonproliferative diabetic retinopathy: a preliminary feasibility study. Clin Invest Med 1990; 13(5): 287-292.

47 Kirkegaard C et al. Effect of one year continuous subcutaneous infusion of a somatostatin analogue, octreotide, on early retinopathy, metabolic control and 
thyroid function in Type I (insulin-dependent) diabetes mellitus. Acta Endocrinol (Copenhagen) 1990; 122(6): 766-772.

48 Grant MB et al. The efficacy of octreotide in the therapy of severe nonproliferative and early proliferative diabetic retinopathy: a randomized controlled study. Diabetes Care 2000; 23(4): 504-509.

49 Kuijpers RW, Baarsma S, van Hagen PM. Treatment of cystoid macular edema with octreotide. N Engl J Med 1998; 338(9): 624-626.

50 Luettke BLG, Boehm BO, Lang GK. Influence of a Somatostatin analogue on the course of persisting proliferative diabetic retinopathy after laser treatment. Invest Ophthalmol Vis Sci 1998; 39(S674): abstract 3100 .

51 Growth Hormone Antagonist for Proliferative Diabetic Retinopathy Study Group. The effect of a growth hormone receptor antagonist drug on proliferative diabetic retinopathy. Ophthalmology 2001; 108: 226-227.

52 Yeo TK et al. Glycosylation is essential for efficient secretion but not for permeability-enhancing activity of vascular permeability factor (vascular endothelial growth factor). Biochem Biophys Res Commun 1991; 179(3): 1568-1575

53 Tolentino MJ et al. Intravitreous injections of vascular endothelial growth factor produce retinal ischemia and microangiopathy in an adult primate. Ophthalmology 1996; 103(11): 1820-1828.

54 Xia $\mathrm{P}$ et al. Characterization of vascular endothelial growth factor's effect on the activation of protein kinase C, its isoforms, and endothelial cell growth. J Clin Invest 1996; 98(9): 2018-2026.

55 Preclinical and phase 1A clinical evaluation of an anti-VEGF pegylated aptamer (EYE001) for the treatment of exudative age-related macular degeneration. Retina 2002; 22(2): 143-152.

56 Donnelly R. Protein kinase C: a novel therapeutic target in microvascular complications. Mod Diabetes Manage 2000; 1(1): 13-16.

57 Koya D, King GL. Protein kinase C activation and the development of diabetic complications. Diabetes 1998; 47(6): 859-866.

58 Kirk Ways D, Protein kinase C beta and chronic complications of diabetes. Diabetes Rev Int 1998; 7(4): 9-12.

59 Koya D et al. d-alpha-tocopherol treatment prevents glomerular dysfunctions in diabetic rats through inhibition of protein kinase C-diacylglycerol pathway. Biofactors 1998; 7(1-2): 69-76.

60 Ishii $\mathrm{H}$ et al. Amelioration of vascular dysfunctions in diabetic rats by an oral PKC beta inhibitor. Science 1996; 272(5262): 728-731.

61 Aiello LP et al. Vascular endothelial growth factor-induced retinal permeability is mediated by protein kinase $C$ in vivo and suppressed by an orally effective beta-isoform-selective inhibitor. Diabetes 1997; 46(9): 1473-1480.

62 Danis RP et al. Inhibition of intraocular neovascularization caused by retinal ischemia in pigs by PKCbeta inhibition with LY333531. Invest Ophthalmol Vis Sci 1998; 39(1): 171-179.

63 Ulrich P, Cerami A. Protein glycation, diabetes, and aging. Recent Prog Horm Res 2001; 56: 1-21.

64 Bucala R, Model P, Cerami A. Modification of DNA by reducing sugars: a possible mechanism for nucleic acid aging and age-related dysfunction in gene expression. Proc Natl Acad Sci USA 1984; 81(1): 105-109.
65 Howard EW et al. Cellular contraction of collagen lattices is inhibited by nonenzymatic glycation. Exp Cell Res 1996; 228(1): 132-137.

66 Paget $\mathrm{C}$ et al. Modification of enzymatic antioxidants in retinal microvascular cells by glucose or advanced glycation end products. Free Radical Biol Med 1998; 25(1): 121-129.

67 Stitt AW. Advanced glycation: an important pathological event in diabetic and age related ocular disease. $\mathrm{Br} J$ Ophthalmol 2001; 85(6): 746-753.

68 Ellis EN, Good BH. Prevention of glomerular basement membrane thickening by aminoguanidine in experimental diabetes mellitus. Metabolism 1991; 40(10): 1016-1019.

69 Soulis-Liparota $\mathrm{T}$ et al. Retardation by aminoguanidine of development of albuminuria, mesangial expansion, and tissue fluorescence in streptozocin-induced diabetic rat. Diabetes 1991; 40(10): 1328-1334.

70 Bach LA et al. Aminoguanidine ameliorates changes in the IGF system in experimental diabetic nephropathy. Nephrol Dial Transplant 2000; 15(3): 347-354.

71 Hammes HP et al. Aminoguanidine treatment inhibits the development of experimental diabetic retinopathy. Proc Natl Acad Sci USA 1991; 88(24): 11555-11558.

72 Roufail E et al. Depletion of nitric oxide synthase-containing neurons in the diabetic retina: reversal by aminoguanidine. Diabetologia 1998; 41(12): 1419-1425.

73 Huijberts MS et al. Aminoguanidine treatment increases elasticity and decreases fluid filtration of large arteries from diabetic rats. J Clin Invest 1993; 92(3): 1407-1411.

74 Brown CD et al. Correction of erythrocyte deformability defect in ALX-induced diabetic rabbits after treatment with aminoguanidine. Diabetes 1993; 42(4): 590-593.

75 Panagiotopoulos $\mathrm{S}$ et al. Aminoguanidine has an antiatherogenic effect in the cholesterol-fed rabbit. Atherosclerosis 1998; 136(1): 125-131.

76 Kihara $\mathrm{M}$ et al. Aminoguanidine effects on nerve blood flow, vascular permeability, electrophysiology, and oxygen free radicals. Proc Natl Acad Sci USA 1991; 88(14): 6107-6111.

77 Miyauchi $Y$ et al. Slowing of peripheral motor nerve conduction was ameliorated by aminoguanidine in streptozocin-induced diabetic rats. Eur J Endocrinol 1996; 134(4): 467-473.

78 Kern TS, Engerman RL. Pharmacological inhibition of diabetic retinopathy: aminoguanidine and aspirin. Diabetes 2001; 50(7): 1636-1642.

79 Vasan $\mathrm{S}$ et al. An agent cleaving glucose-derived protein crosslinks in vitro and in vivo. Nature 1996; 382(6588): 275-278.

80 Wolffenbuttel BH et al. Breakers of advanced glycation end products restore large artery properties in experimental diabetes. Proc Natl Acad Sci USA 1998; 95(8): 4630-4634.

81 Asif $\mathrm{M}$ et al. An advanced glycation endproduct cross-link breaker can reverse age-related increases in myocardial stiffness. Proc Natl Acad Sci USA 2000; 97(6): 2809-2813.

82 Nakamura $S$ et al. Progression of nephropathy in spontaneous diabetic rats is prevented by OPB-9195, a novel inhibitor of advanced glycation. Diabetes 1997; 46(5): 895-899.

83 Baynes JW, Thorpe SR. Role of oxidative stress in diabetic complications: a new perspective on an old paradigm. Diabetes 1999; 48(1): 1-9.

84 Vlassara H, Palace MR. Diabetes and advanced glycation endproducts. J Intern Med 2002; 251(2): 87-101.

85 Verdejo C et al. Lipid peroxidation in proliferative vitreoretinopathies. Eye 1999; 13(Part 2): 183-188. 
86 Chung TW, Yu JJ, Liu DZ. Reducing lipid peroxidation stress of erythrocyte membrane by alpha-tocopherol nicotinate plays an important role in improving blood rheological properties in type 2 diabetic patients with retinopathy. Diabetic Med 1998; 15(5): 380-385.

87 Kunisaki $\mathrm{M}$ et al. Prevention of diabetes-induced abnormal retinal blood flow by treatment with d-alpha-tocopherol. Biofactors 1998; 7(1-2): 55-67.
88 Granado F et al. Carotenoids, retinol and tocopherols in patients with insulin-dependent diabetes mellitus and their immediate relatives. Clin Sci (Lond) 1998; 94(2): 189-195.

89 Dodson PM. Oral agents for retinopathy: from graveyard to hope? Mod Diabetes Manage 2001; 2(3): 1 (leading article)

90 Ferris FL, Davis MD, Aiello LM. Treatment of diabetic retinopathy. N Engl J Med 1999; 341: 667-678. 